\title{
Anti-tumor effect of apicidin on Ishikawa human endometrial cancer cells both in vitro and in vivo by blocking histone deacetylase 3 and 4
}

\author{
MEE YOUNG AHN ${ }^{1}$, HAE YOUNG CHUNG ${ }^{1}$, WAHN SOO $\mathrm{CHOI}^{2}$, \\ BYUNG MU LEE ${ }^{3}$, SUNGPIL YOON ${ }^{4}$ and HYUNG SIK KIM ${ }^{1}$ \\ ${ }^{1}$ College of Pharmacy, Pusan National University, San 30, Jangjeon-dong, Geumjeung-gu, Busan 609-735; \\ ${ }^{2}$ Department of Immunology, College of Medicine, Konkuk University, Chungju-Si 380-701; ${ }^{3}$ Division \\ of Toxicology, School of Pharmacy, Sungkyunkwan University, Suwon; ${ }^{4}$ Research Institute, \\ National Cancer Center, 809 Madu 1-dong, Ilsan-gu, Goyang-si, Gyeonggi-do, Korea
}

Received August 28, 2009; Accepted October 12, 2009

DOI: 10.3892/ijo_00000483

\begin{abstract}
Histone deacetylase (HDAC) inhibitors are a new class of anticancer agents that act by inhibiting cancer cell proliferation and inducing apoptosis both in vitro and in vivo. This study examined the anti-tumor effect of apicidin on human endometrial cancer Ishikawa cells in an animal model by inhibiting specific HDAC expression. Nude mice were injected subcutaneously (s.c.) with Ishikawa cells, and the levels of cell proliferation and apoptosis were measured in the tumor tissues after an apicidin treatment. The expression patterns of a specific HDAC class by apicidin were measured in Ishikawa endometrial cancer both in vitro and in vivo. The tumor volume and weight were measured after the apicidin treatment. Apicidin significantly increased the acetylated histone $\mathrm{H} 3$ levels in an Ishikawa cells in vitro culture but the levels of HDAC3 and HDAC4 expression were significantly decreased. Apicidin suppressed the tumor growth of transplanted Ishikawa cells, the expression of proliferative cell nuclear antigen (PCNA) and vascular endothelial growth factor (VEGF) in tumor xenograft model, respectively. The inhibitory effect of apicidin on tumor growth was mediated in part through the down-regulation of HDAC3 and HDAC4. We suggest that apicidin is an effective anti-tumor agent on human endometrial cancer cells, and acts by regulating cell proliferation and apoptosis through the down-regulation of HDAC3 and HDAC4.
\end{abstract}

Correspondence to: Professor Hyung Sik Kim, College of Pharmacy, Pusan National University, San 30, Jangjeon-dong, Geumjeung-gu, Busan 609-735, Korea

E-mail: hkims@pusan.ac.kr

Key words: histone deacetylase inhibitor, apicidin, endometrial cancer, apoptosis, xenograft model

\section{Introduction}

Histone deacetylases (HDACs) play a key role in the epigenetic regulation of genes by catalyzing the removal of acetyl groups, stimulating chromatin condensation and promoting transcriptional repression (1). The loss of expression of tumor suppressors, cell-cycle inhibitors and differentiation factors or apoptosis inducers by histone hypo-acetylation would be advantageous to a cancer cell (2). The overexpression of HDAC enzymes has been observed in many primary human cancer tissues, including stomach, colon, breast, and prostate cancer (3-6). Inhibitors of HDAC enzymes have emerged as new targets for cancer therapy (7-10).

The inhibition of HDAC activity can reverse epigenetic silencing that is commonly observed in cancer, and various HDAC inhibitors have been developed for cancer therapy (11). Several types of HDAC inhibitors with a variety of chemical structures have been identified from natural and synthetic sources. HDAC inhibitors can be divided into five structural classes, such as hydroxamic acid-derived compounds, cyclic peptides, short-chain fatty acids, benzamides and ketones $(12,13)$. Although HDAC inhibitors show a broad spectrum of anti-tumor activities, the concentration of HDAC inhibitor and time course of the experiments were slightly different depending on the cell type both in vitro and in vivo $(14,15)$

Apicidin, a cyclic tetrapeptide isolated from Fusarium $s p$., was first reported to be a reversible inhibitor of the in vitro development of Apicomplexan parasites. Apicidin acts by inhibiting parasitic (including Plasmodium species) HDAC enzyme and was only later shown to have anti-proliferative and cyto-differentiation activity on mammalian cells (16). Apicidin inhibits cell proliferation in several human cancer cell lines, including leukemia, cervical cancer, gastric cancer and breast cancer (17). It was previously reported that apicidin induces the acetylation of histones and has anti-tumor properties on endometrial cancer cells by inducing selectively the genes related to cell cycle arrest and apoptosis in vitro (18). However, it is still unclear if apicidin acts selectively against 
the various HDAC isoforms in human endometrial cancer cells and the anti-tumor effect on Ishikawa endometrial cancer cells in vivo has not been reported.

This study examined the expression of specific HDAC isoforms and the selective HDAC inhibitory effects of apicidin in Ishikawa cancer cells both in vitro and in vivo. In order to further examine the anti-tumor effect of apicidin on Ishikawa cancer cells, Balb/c nude mice bearing an Ishikawa endometrial cancer xenograft were treated with apicidin, and its effect of tumor growth was monitored. The effect of apicidin on inducing apoptosis and HDAC inhibition was analyzed in vivo.

\section{Materials and methods}

Chemicals. Unless stated otherwise, apicidin $[\mathrm{cyclo}(\mathrm{N}-\mathrm{O}-$ methyl-L-tryptophanyl-L-isoleucinyl-D-pipecolinyl-L-2-amino8-oxodecanoyl)] and all other chemicals were purchased from Sigma (St. Louis, MO, USA). Apicidin was dissolved in sterile dimethyl sulfoxide (DMSO) to generate a $5 \mathrm{mM}$ stock solution, and stored at $-80^{\circ} \mathrm{C}$. Subsequent dilutions were made in RPMI-1640 (Gibco, Rockville, MD, USA).

Cell culture. The Ishikawa cancer cells were kindly provided by Dr Jacques Simard (CHUL Research Center, Quebec, Canada). The cells were maintained as monolayers at $37^{\circ} \mathrm{C}$ in DMEM (Gibco) containing 10\% heat-inactivated fetal bovine serum (FBS, Gibco) and 1\% penicillin/streptomycin (Gibco) in an atmosphere containing $5 \% \mathrm{CO}_{2} /$ air. For the apicidin treatment, the cells were plated for $48 \mathrm{~h}$ in DMEM containing $10 \%$ FBS. The medium was then changed to DMEM containing $5 \%$ charcoal-dextran treated FBS (CD-FBS, Gibco) with various concentrations of apicidin.

Cell viability. Cell growth was quantified using 3-(4,5dimethylthiazol-2-yl)-2,5-diphenyltetrazolium bromide (MTT, $5 \mathrm{mg} / \mathrm{ml}$ ) dissolved in phosphate-buffered saline (PBS). The cultures were initiated in 96-well plates at a density of $1 \times 10^{4}$ cells per well. The cells were allowed to attach for $48 \mathrm{~h}$ before being exposed to apicidin. At the end of the treatment period, $15 \mu \mathrm{l}$ of the MTT reagent was added to each well. After $4 \mathrm{~h}$ incubation at $37^{\circ} \mathrm{C}$, the supernatant was aspirated, and the formazan crystals were dissolved in $100 \mu 1 \mathrm{DMSO}$ at $37^{\circ} \mathrm{C}$ for $10 \mathrm{~min}$ with gentle agitation. The absorbance per well was measured at $540 \mathrm{~nm}$ using a VERS Amax Microplate Reader (Molecular Devices Corp., USA).

Western blot analysis and antibodies. The cells were then washed twice with PBS, suspended in a lysis buffer [50 mM Tris ( $\mathrm{pH} 8.0$ ), $150 \mathrm{mM} \mathrm{NaCl}, 0.1 \% \mathrm{SDS}, 0.5 \%$ sodium deoxycholate, $1 \%$ NP40, phenylmethylsulfonyl fluoride $100 \mu \mathrm{g} / \mathrm{ml}$, aprotinin $2 \mu \mathrm{g} / \mathrm{ml}$, pepstatin $\mu \mathrm{g} / \mathrm{ml}$, and leupeptin $10 \mu \mathrm{g} / \mathrm{ml}$, and placed on ice for $30 \mathrm{~min}$. The suspension was collected after centrifugation at $15,000 \mathrm{x}$ g for $15 \mathrm{~min}$ at $4^{\circ} \mathrm{C}$. The animal tissues were homogenized in a lysis buffer containing $50 \mathrm{mM}$ Tris- $\mathrm{HCl}$ (pH 7.5), $150 \mathrm{mM} \mathrm{NaCl}, 1 \mathrm{mM}$ EDTA, $2.5 \mathrm{mM}$ EGTA, $10 \%$ glycerol, $1 \mathrm{mM}$ dithiothreitol, $100 \mathrm{mM}$ phenylmethyl sulfonyl fluoride, $10 \mu \mathrm{g} / \mathrm{ml}$ aprotinin, and $10 \mu \mathrm{g} / \mathrm{ml}$ leupeptin. After incubation for $30 \mathrm{~min}$ at $4^{\circ} \mathrm{C}$, the homogenate was centrifuged at $16,000 \mathrm{x} \mathrm{g}$ for $20 \mathrm{~min}$ at

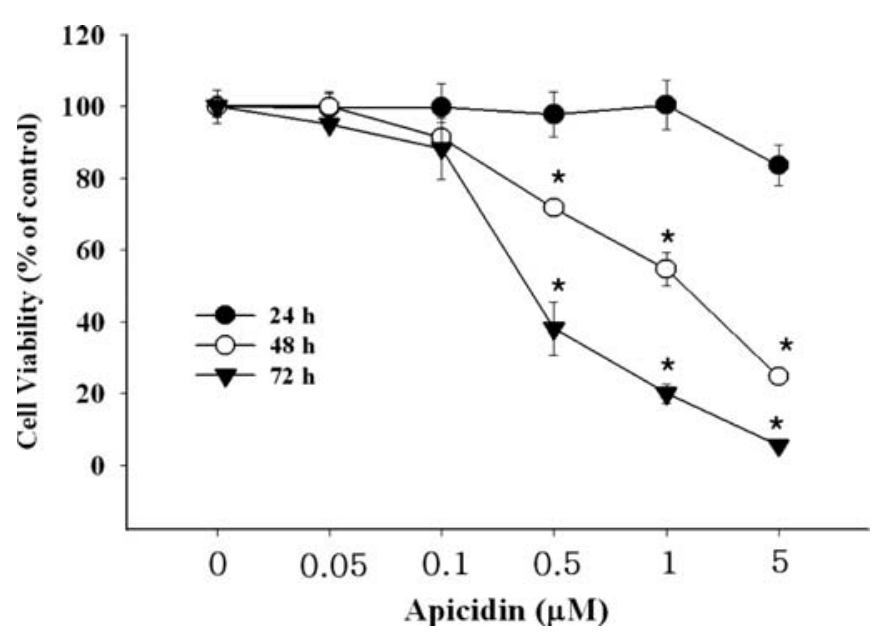

Figure 1. Effect of apicidin on Ishikawa cells viability. The cells were treated with apicidin at various concentrations (0.01-10 $\mu \mathrm{M})$ for the indicated times. The level of proliferation was measured using an MTT assay. The percentage of viable cells was calculated as the ratio of the treated to control cells. The data are expressed as the mean \pm SD of three independent experiments. ${ }^{*} \mathrm{P}<0.05$ as determined by a Student's t-test.

$4^{\circ} \mathrm{C}$, and the supernatant was collected to determine the protein concentration. The protein concentrations were quantified using a Bio-Rad protein assay reagent (Bio-Rad Laboratories) according to the manufacturer's protocol. The whole cell lysates and tumor homogenates $(50 \mu \mathrm{g})$ were resolved on SDS-polyacrylamide gel in an 8-12\% gel, transferred to a polyvinylidene difluoride (PVDF) membrane (NEN life Science, Boston, USA), and probed sequentially with the antibodies against acetylated $\mathrm{H} 3$ (Upstate, Lake Placid, NY, USA), p21 (BD Biosciences, San Jose, CA, USA), HDAC1 (Cell Signaling, MA, USA), HDAC2, HDAC3, HDAC4, HDAC5, HDAC7 (all from Cell Signaling) and $\beta$-actin antibody (Sigma). The blots were developed using an enhanced chemiluminescence (ECL) kit (Amersham, Cardiff, UK).

In vivo tumor xenograft model. Six-week-old female nude mice (BALB-c nu/nu, Charles River Lab. Inc., Wilmington, MA, USA) were housed under controlled temperature $\left(22 \pm 2^{\circ} \mathrm{C}\right)$ and lighting ( $12 \mathrm{~h} \mathrm{light/dark} \mathrm{cycle)} \mathrm{contains} \mathrm{in} \mathrm{filtered-air}$ laminar-flow cabinets and manipulated using aseptic procedures. All animal procedures were approved by the Korea Medical Experimental Animal Care Commission (Daejon, Korea), prior to experimentation. Ishikawa cells $\left(2 \times 10^{7}\right)$ in $0.1 \mathrm{ml}$ serum-free medium containing $50 \%$ Matrigel were injected subcutaneously (s.c.) into the upper flank of each nude mouse. When the tumor size reached $200 \mathrm{~mm}^{3}$, mice were randomized to two groups $(\mathrm{n}=5)$. Apicidin $5 \mathrm{mg} / \mathrm{kg}$ was administered intraperitoneally to the mice daily for 21 days and the control mice were administered $0.1 \%$ DMSO in the same manner. The tumor sizes were measured with calipers and their volumes calculated using the standard formula: width $^{2} \mathrm{x}$ length $\mathrm{x} 0.52$. The body weights were recorded prior to dosing. The mice were sacrificed at the end of the treatment period. The tumor tissue was excised, weighed, frozen in liquid nitrogen, and stored at $-80^{\circ} \mathrm{C}$ until needed. In addition, the tumor tissue was fixed in $4 \%$ paraformaldehyde, and 


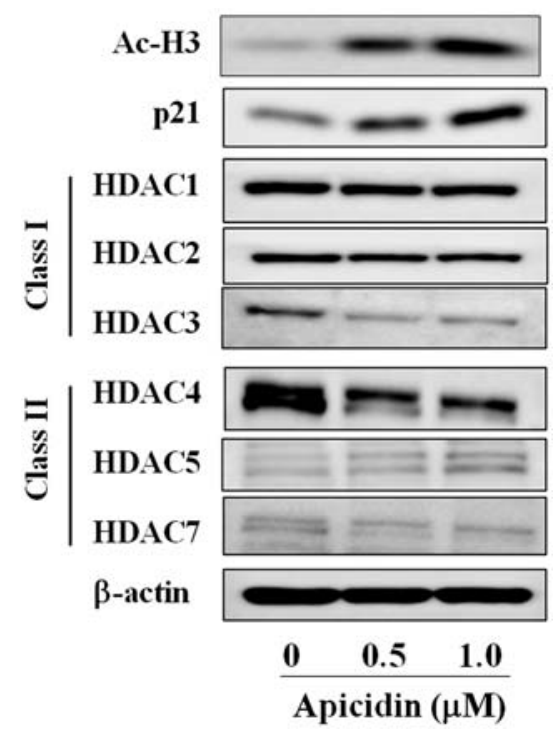

Figure 2. Effect of apicidin on HDAC isoenzymes levels in Ishikawa cells. Ishikawa cells were treated with apicidin at indicated concentrations for $48 \mathrm{~h}$. Total cell lysates were prepared and the protein was subjected to SDS-PAGE followed by Western blot analysis and chemiluminescent detection. The control cells were treated with the vehicle alone. Western blot analysis was performed with the HDACs isoenzymes, acetylated $\mathrm{H} 3$ and p21 antibodies. The amount of protein was normalized by a comparison to the $\beta$-actin levels. Representative bands from three independent experiments are shown.

embedded in paraffin. Paraffin sections $(5 \mu \mathrm{m})$ were used for histological analysis. The tumor sections were taken for hematoxylin and eosin (H\&E) staining and viewed under an optical microscope (magnification, x200, Leica, Wetzler,
Germany). The images recorded by a digital camera (Olympus Optical Co. Ltd., Tokyo, Japan).

Immunohistochemistry. Paraffin-embedded tissues were used to identify the proliferating cell nuclear antigen (PCNA) and vascular endothelial growth factor (VEGF). Deparaffinization was achieved with xylene followed by a descending series of ethanol concentrations. Antigen retrieval was carried out in a microwave-heated citrate buffer ( $\mathrm{pH} \mathrm{6.0)}$ for $20 \mathrm{~min}$. The endogenous peroxidases were blocked with $3 \% \mathrm{H}_{2} \mathrm{O}_{2} /$ methanol for $15 \mathrm{~min}$ at room temperature. The non-specific epitopes were blocked with $1 \%$ normal goat serum for $30 \mathrm{~min}$ at room temperature. The tumor sections were incubated overnight at $4^{\circ} \mathrm{C}$ with PCNA (1:200, Lab Vision Corp., CA, USA) and VEGF (1:130, Santa Cruz, CA, USA) antibodies. The immunoreactions were visualized using a streptavidin-biotin complex method followed by a diaminobenzidine reaction (Zymed, USA). The tumor sections were counterstained with hematoxylin in order to visualize the nuclei. The immunoreactions were viewed under an optical microscope (magnification, x400, Leica), and the images recorded on a digital camera (Olympus Optical Co. Ltd.).

TUNEL assay. The level of apoptosis was determined using an ApopTag in situ apoptosis detection kit (Chemicon International, Temecula, CA) according to the manufacturer's protocol. To determine TUNEL expression in tissue sections, the number of apoptotic events was counted in ten random fields by confocal laser scanning microscopy (TCS SP2, Leica) at a magnification, $\mathrm{x} 400$ and that number was divided by the total number of cells per field.
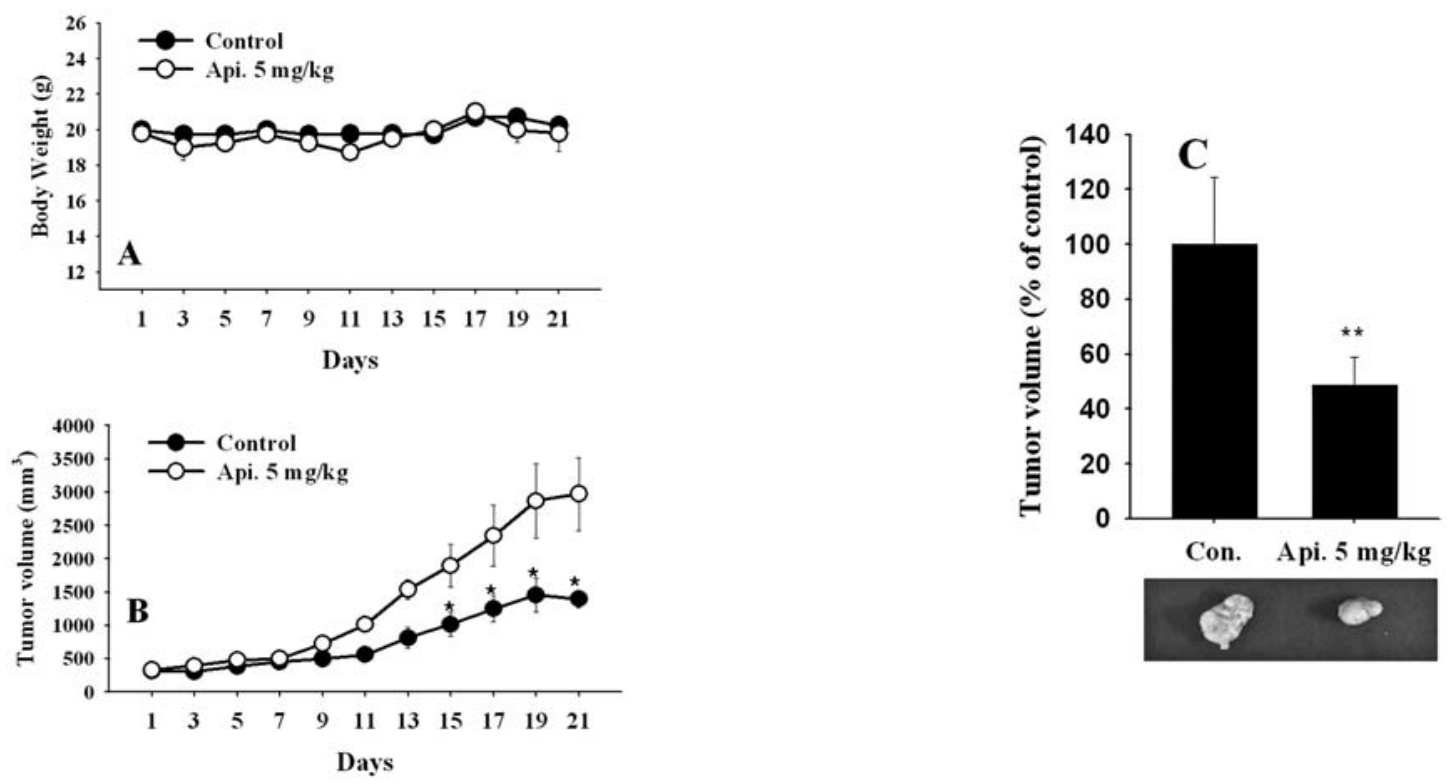

Figure 3. Effect of apicidin on the growth of Ishikawa endometrial cancer xenograft model. (A) An assessment of the body weight between the treated and control mice during entire experimental period (21 days). The body weight of the mice in different groups was recorded every alternate day. (B) Relative tumor volumes in mice inoculated with Ishikawa cancer cells $(n=5)$. The relative tumor volumes of the mice treated with apicidin and the vehicle treated controls. The tumor volumes were measured and transformed subsequently to the relative tumor volume, as detailed in Materials and methods. (C) In situ appearance of tumors in control and apicidin-treated mice. i) Grafted tumors in the vehicle treated control nude mice, ii) grafted tumors in the vehicle treated control nude mice, iii) the gross size of the tumors from the vehicle treated control group, iv) the gross size of the tumors from the vehicle treated control group. Final tumor volumes of Ishikawa xenograft. The data are reported as mean $\pm \mathrm{SD}$ of five animals. ${ }^{*} \mathrm{P}<0.05$, ${ }^{* *} \mathrm{P}<0.01$ as determined by a Student's t-test compared to the control group. 


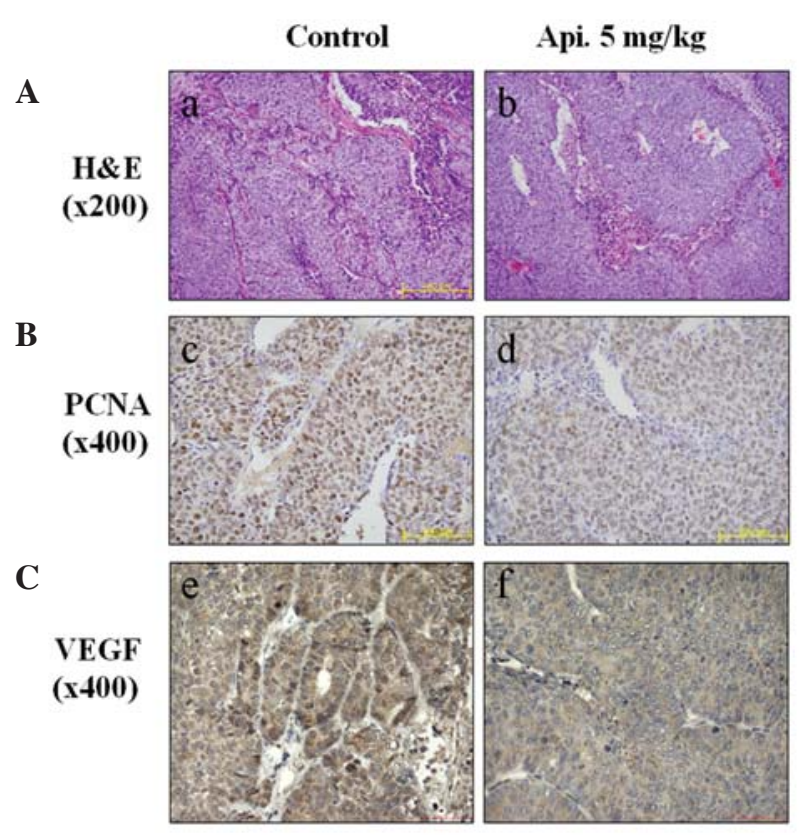

Figure 4. Effect of apicidin on growth and angiogenesis inhibition in Ishikawa tumor xenograft. (A) Optical microscope images of H\&E stained sections of tumors. (a) Vehicle treated control group and (b) apicidin treated group. The tissues were harvested, processed, stained with $\mathrm{H} \& \mathrm{E}$ and viewed at a magnification, $\mathrm{x} 200$ for a microscopic evaluation at the end of the study. Scale bar=100 $\mu \mathrm{M}$. (B) Representative results of PCNA staining in tumors (c) The vehicle-treated control group and (d) apicidin-treated group. The rate of proliferation was measured by immunohistochemical staining using the PCNA antibody. Photographs were taken under a magnification, x400. Scale bar=200 $\mu \mathrm{M}$. (C) Representative results of VEGF staining in tumors. (e) The vehicle-treated control group and (f) apicidin-treated group. The rate of proliferation rate was measured by immunohistochemical staining using the VEGF antibody. The photographs were taken under magnification, $\mathrm{x} 400$. Scale bar $=200 \mu \mathrm{M}$
Statistical analysis. All the data are represented as the mean \pm SD. The significance was determined using a paired Student's t-test. A * $<<0.05$ and ${ }^{* *} \mathrm{p}<0.01$ were considered statistically significant.

\section{Results}

Apicidin inhibits cell proliferation and induces the acetylated histone levels in Ishikawa cancer cells. Previously, it was suggested apicidin inhibits the proliferation of a variety of human cancer cell lines including SK-OV-3 and Ishikawa cells $(18,19)$. In order to confirm apicidin-induced cytotoxicity on endometrial cancer cells, the cells were treated with varying doses of the drug for 24,48 or $72 \mathrm{~h}$, and the cell viability was measured using an MTT assay. Apicidin inhibited the proliferation of Ishikawa cancer cells in a concentration-dependent manner, and a significant inhibition was observed even at the low concentration $(0.5 \mu \mathrm{M})$ after $72 \mathrm{~h}$ of treatment (Fig. 1). This dose-response curve suggests that the $\mathrm{IC}_{50}$ value of apicidin is $0.41 \mu \mathrm{M}$ at $72 \mathrm{~h}$. Moreover, the apicidin treatment reduced significantly the levels of the HDAC 3 and HDAC4 proteins in Ishikawa cells, particularly HDAC4 (Fig. 2). In particular, apicidin significantly increased the expression level of p21 in a dose-dependent manner (Fig. 2).

Tumor xenograft model. Athymic nude mouse model using tumor xenografts were established to evaluate the anti-tumor potential of apicidin on Ishikawa cancer cells in vivo. No systemic toxicity including the body weight changes, diarrhea, or other apparent adverse effects were observed in the animals throughout the study period (Fig. 3A). The tumors in the

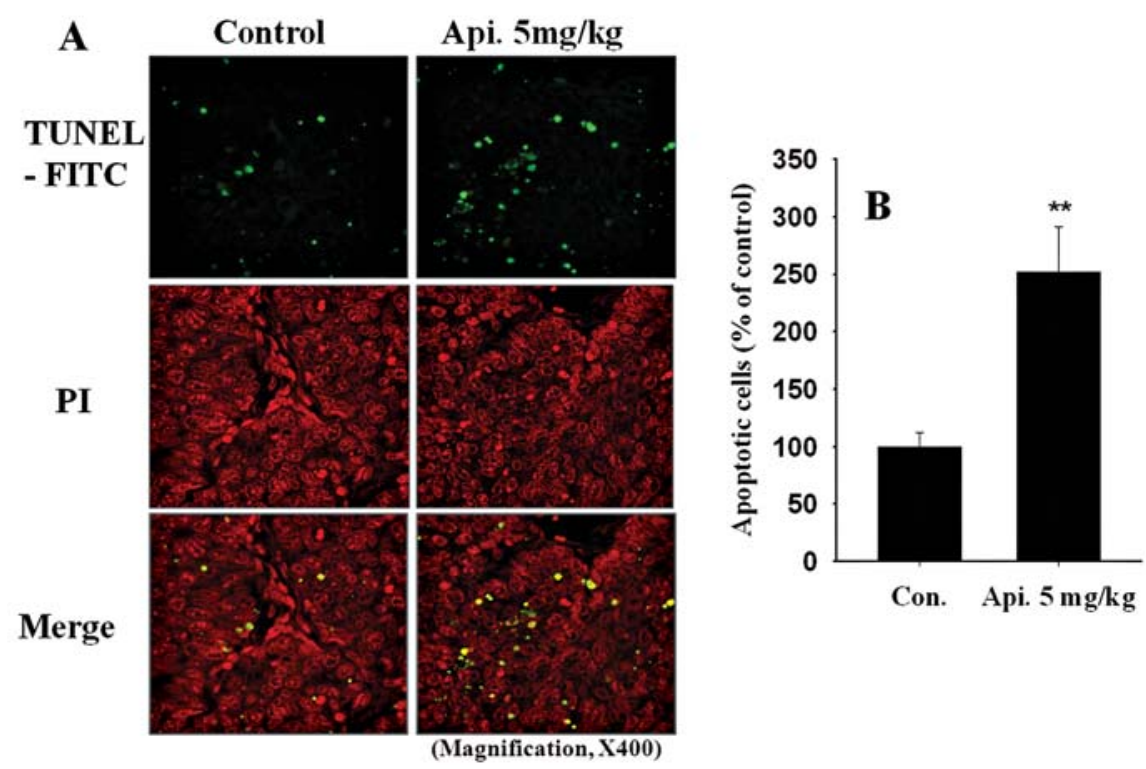

Figure 5. Effect of apicidin on Ishikawa tumor cell apoptosis in tumor xenografts. Immunohistochemical staining was carried out on the apicidin-treated and control tumors using TUNEL assay. (A) Representative photos of TUNEL staining in the vehicle treated control group and apicidin treated group. The apoptotic cells were detected using ApopTag in situ apoptosis detection kits and the results are shown as a confocal microscopy image (magnification, x400). (B) Comparison of the TUNEL-positive cells in the apicidin-treated and control groups. The number of apoptotic cells in ten random fields at a magnification, $\mathrm{x} 400$ was counted, and the number was divided by the total number of cells per field. The data are reported as the mean \pm SD of two independent experiments. ${ }^{* *} \mathrm{P}<0.01$ as determined by Student's t-test compared to controls. 
A

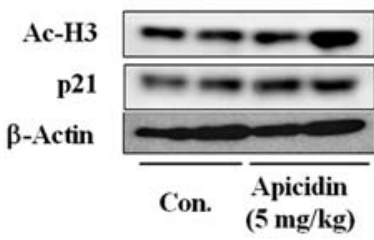

$\mathbf{C}$

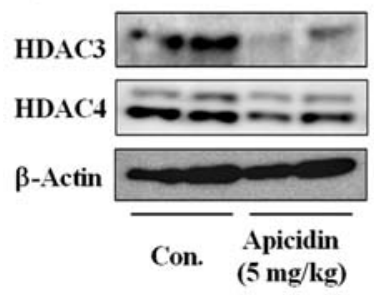

B

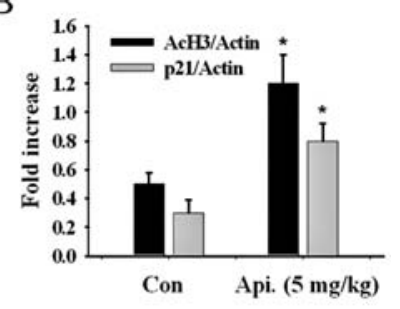

D

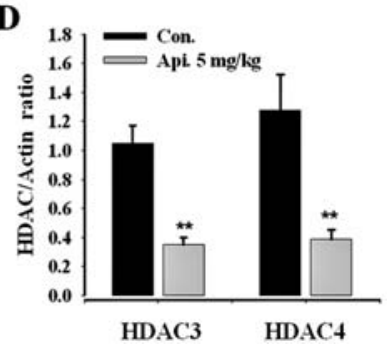

Figure 6. The expression on the acetylation-related protein levels in Ishikawa xenograft. (A) Western blot analysis of acetylated H3 and p21 in the homogenates of two representative Ishikawa tumors from each treatment group. (B) Relative expression levels of acetylated histone 3 and p21 in the vehicle- and apicidin-treated group mice. (C) Western blot analysis of HDAC3 and HDAC4 in the homogenates of two representative Ishikawa tumors from each treatment group. (D) Relative levels of HDAC3 and HDAC4 expression in the vehicle- and apicidin-treated group mice. The amount of protein was normalized by a comparison to the $\beta$-actin levels. The data are reported as the mean \pm SD of two bands. ${ }^{*} \mathrm{P}<0.05,{ }^{* *} \mathrm{P}<0.01$ as determined by the Student's t-test compared to the controls.

control group grew continuously during the experimental period, whereas tumor volume in the apicidin-treated mice increased slightly after the treatment and was then suppressed at later time points. Significant inhibition of tumor growth was observed starting from day 15 after the apicidin treatment (Fig. 3B). Apicidin (5 mg/kg) significantly inhibited tumor growth up to $53 \%$ relative to the control group. The average tumor volume in the apicidin $5 \mathrm{mg} / \mathrm{kg}$ treated mice 21 days after initiating treatment was $1,400 \mathrm{~mm}^{3}$, whereas the tumor volume of the control mice at the same period was $2,950 \mathrm{~mm}^{3}$ (Fig. 3C).

PCNA and VEGF expression in tumor xenograft model. The histological changes between the apicidin-treated and control group were examined using H\&E staining in paraffinembedded tumor sections. As shown in Fig. 4A, the tumor tissues treated with apicidin had larger areas of necrosis and fewer blood vessels than the control. The anti-tumor effects of apicidin in vivo model were investigated by examining the expression of PCNA, a cell proliferation marker, and VEGF, an angiogenetic factor by immunohistochemistry in paraffinembedded tumor sections. The percentage of cancer cells with a positive stained nucleus for PCNA was much higher in the control group than apicidin-treated group (Fig. 4B). Apicidin treatment decreased the expression of VEGF in Ishikawa xenograft as shown in Fig. 4C. These results demonstrate that the in vitro effect of apicidin on the inhibition of tumor cell proliferation and blood vessels correlates with the anti-cancer activity of apicidin in vivo.

Apoptosis induction in tumor xenograft model. The antitumor effect of apicidin was studied through the induction of

apoptosis in Ishikawa cells in vitro (18). Apoptotic cell death was next examined by a TUNEL assay in paraffin-embedded tumor sections. Apicidin significantly increased the TUNEL positive apoptotic cell population 2.5-fold compared to those in the vehicle-treated control with $\mathrm{p}<0.01$ (Fig. 5). These results show that the in vitro effect of apicidin on the induction of apoptosis is associated with the anti-cancer activity of apicidin in vivo.

Expression of acetylated $\mathrm{H} 3$ and HDACs in Ishikawa cell xenografts. To confirm the histone acetylative effect of apicidin in vivo, the levels of acetylated $\mathrm{H} 3$ expression in Ishikawa cells xenograft tumor tissue were measured by Western blot analysis. As shown in Fig. 6A, the tumors treated with apicidin showed up-regulation of acetylated $\mathrm{H} 3$ and p21 expression compared to those in the vehicle-treated controls. The inhibitory effect of apicidin on the specific HDAC isoforms in tumor xenograft was next investigated. The expression of HDAC3 and HDAC4 was lower in the apicidin-treated Ishikawa cells in vitro. Therefore, the expression of HDAC3 and HDAC4 in Ishikawa cell xenograft tumor tissue was examined by Western blotting. The levels of HDAC3 and HDAC4 expression were downregulated in the apicidin-treated tumor tissues. The relative expression levels of HDAC3 and HDAC4 were reduced by 65 and $59 \%$ compared to those in the vehicle-treated controls, respectively (Fig. 6B). This shows that the inhibitory effect of tumor growth correlates with the inhibition of HDAC expression in vivo.

\section{Discussion}

Endometrial cancer is one of the most common gynecological malignancies and develops in approximately 142,000 women in worldwide with an estimated 42,000 deaths (20). There are two major types (I and II) of endometrial cancer with specific features and different changes in a genetic setting (21). HDAC inhibitors are an emerging class of targeted cancer therapeutics, and may have an important impact on the treatment of both types I and II endometrial carcinoma (22-24). Apicidin is a cyclic peptide group of HDAC inhibitors, and accounts for the in vitro high acetylation level of histones, which correlates with an increase in the transcription of various cancer-related genes (25). It was reported previously that an apicidin treatment increased the levels of acetylated histone and induced cell cycle arrest and apoptosis in Ishikawa endometrial cancer cells (18).

Based on the in vitro anticancer effect of apicidin, the anti-tumor effect of apicidin on Ishikawa endometrial cancer cells was evaluated in the tumor xenograft model using nude mice. Apicidin induced 53\% inhibition of tumor growth in the Ishikawa cell xenografts compared to the vehicle alone. These results suggest that apicidin not only inhibits Ishikawa cell growth in vitro but also has a profound effect on the growth inhibition of endometrial tumors in vivo. In order to explore the possible mechanisms underlying tumor growth inhibition evidenced in the apicidin treatment, its effects on tumor cell proliferation was first examined by calculating the proliferative index PCNA on the tumors collected at necropsy from all experiments. The tumor sizes correlated well with 
the expression of PCNA-stained nuclei, as observed by immunohistochemistry. Furthermore, the number of PCNA positive cells was significantly lower in the apicidin-treated group than the vehicle group of mice.

Angiogenesis is essential for the development, growth and metastasis of tumors (26). The VEGF stimulates angiogenesis by promoting endothelial cell proliferation, and its expression is regulated by the HDAC activity (27). A previous study reported that the levels of VEGF were elevated significantly in endometrial cancer (28). In the present study, apicidin reduced the levels of VEGF significantly in the apicidin-treated group compared to the vehicle group, which showed increased levels of VEGF. This suggests that apicidin can also induce the anti-angiogenic effects in endometrial cancer development in tumor xenograft model.

Tumor cells deprived of nutrients and survival factors due to the loss of an adequate vasculature may then undergo apoptosis (29). Our previous study revealed that apicidin can induce apoptosis in endometrial cancer cells (18). Therefore, a TUNEL assay was performed to determine if apicidin induces apoptosis in vivo. A significantly higher percentage of TUNEL-positive cells were observed in the apicidin treated tumors than in the controls. These results suggest that the induction of apoptosis by apicidin may also be a mechanism for the suppression of Ishikawa xenograft model.

HDAC inhibitors have attracted considerable interest as drug targets in recent years, with several compounds of this class currently in clinical testing $(30,31)$. Despite the broad applications of HDAC inhibitors in cell culture, animal models, and early phase clinical trials, surprisingly little is known about the expression of their targets in cancer tissues (32). The expression of the class I HDACs have been studied in endometrial cancer patients. Each of the class I HDACs was expressed at high levels (HDAC1, 61\%; HDAC2, 95\%; HDAC $3,83 \%$ ) in most endometrial carcinomas samples (23). However, the expression of the other class of HDACs in endometrial carcinomas is largely unknown. Our results showed that the levels of HDAC1, 2, 3 and 4 were expressed strongly in Ishikawa cells, whereas HDAC5, 6 and 7 were expressed weakly.

Most HDAC-inhibitors do not selectively inhibit individual HDAC isoenzymes and an individual HDAC inhibitor might have different effects on different biological systems because there are specific differences in HDAC expression according to the tissue- and cell-type localization and targets (33). This showed that the regulation of specific HDAC inhibition by apicidin may also vary according to the cell type and experimental condition. In this study, the inhibition of specific HDAC subtypes to apicidin was evaluated using an Ishikawa cancer cell xenograft model. The administration of apicidin resulted in the repression of HDAC3 and HDAC4, which was observed in apicidin-treated Ishikawa cells in vitro. HDAC3 expression was reported to be associated with a poor prognosis in a large series of gastric, colorectal and prostate cancer samples (34-36). HDAC3 was up-regulated in colon tumors, and the silencing of HDAC3 expression in colon cancer cell lines resulted in growth inhibition, a decrease in cell survival, and increased apoptosis (37). HDAC4 expression was upregulated in breast cancer samples compared to renal, bladder and colorectal cancer (38). HDAC4 (and HDAC6) were reported to bind to and regulate HIF-1 $\alpha$ transcriptional activity in renal carcinoma cells. Therefore, targeting of these HDACs may be a means of tumor anti-angiogenesis (39). Down-regulating HDAC4 expression by small interfering RNA (siRNA) in HCT116 colon cancer cells induced the inhibition of cell growth and induction of apoptosis in vitro, reduced xenograft tumor growth, and increased p21 transcription (40). These studies provide evidence for the potential role of HDAC3 and HDAC4, and suggest that the repression of HDAC3 and HDAC4 by apicidin might be responsible for its anticancer effect both in vitro and in vivo. Further studies on the individual function of HDAC3 and HDAC4 in mediating the components of apicidin response will be needed to determine the inhibitory mechanism of HDAC inhibitors in endometrial cancer.

In conclusion, apicidin showed anti-tumor activity in vivo, leading to the inhibition of cell proliferation and angiogenesis, and the induction of apoptosis in endometrial cancer. The inhibitory effect of apicidin on tumor growth was mediated in part by the up-regulation of acetylated $\mathrm{H} 3$ and $\mathrm{p} 21$, and the down-regulation of HDAC3 and HDAC4. These results suggest that apicidin is a potential anti-tumor agent against human endometrial cancer.

\section{References}

1. Dokmanovic M, Clarke C and Marks PA: Histone deacetylase inhibitors: overview and perspectives. Mol Cancer Res 5: 981-989, 2007.

2. Glozak MA and Seto E: Histone deaceylase and cancer. Oncogene 26: 5420-5432, 2007.

3. Choi JH, Kwon HJ, Yoon BI, et al: Expression profile of histone deacetylase 1 in gastric cancer tissues. Jpn J Cancer Res 92: 1300-1304, 2001

4. Halkidou K, Gaughan L, Cook S, Leung HY, Neal DE and Robson CN: Upregulation and nuclear recruitment of HDAC1 in hormone refractory prostate cancer. Prostate 59: 177-189, 2004.

5. Kawai H, Li H, Avraham S, Jiang S and Avraham HK: Overexpression of histone deacetylase HDAC1 modulates breast cancer progression by negative regulation of estrogen receptor $\alpha$. Int J Cancer 107: 353-358, 2003.

6. Zhu P, Martin E, Mengwasser J, Schlag P, Janssen KP and Gottlicher M: Induction of HDAC2 expression upon loss of APC in colorectal tumorigenesis. Cancer Cell 5: 455-463, 2004.

7. Johnstone RW: Histone-deacetylase inhibitors: novel drugs for the treatment of cancer. Nat Rev Drug Discov 1: 287-299, 2002.

8. Monneret C: Histone deacetylase inhibitors for epigenetic therapy of cancer. Anticancer Drugs 18: 363-370, 2007.

9. Bolden JE, Peart MJ and Johnstone RW: Anticancer activities of histone-deacetylase inhibitors. Nat Rev Drug Discov 5: 769-784, 2006.

10. Minucci S and Pelicci PG: Histone deacetylase inhibitors and the promise of epigenetic (and more) treatments for cancer. Nat Rev Cancer 6: 38-51, 2006.

11. Carew JS, Giles FJ and Nawrocki ST: Histone deacetylase inhibitors: mechanisms of cell death and promise in combination cancer therapy. Cancer Lett 269: 7-17, 2008.

12. Drummond DC, Noble CO, Kirpotin DB, Guo Z, Scott GK and Benz CC: Clinical development of histone deacetylase inhibitors as anticancer agents. Annu Rev Pharmacol Toxicol 45: 495-528, 2005.

13. Lindemann RK and Johnstone RW: Histone deacetylase inhibitors: promising candidates for chemotherapeutic drugs. Gene Ther Mol Biol 8: 61-74, 2004.

14. Mei S, Ho AD and Mahlknecht U: Role of histone deacetylase inhibitors in the treatment of cancer (Review). Int J Oncol 25: 1509-1519, 2004.

15. Acharya MR, Sparreboom A, Venitz J and Figg WD: Rational development of histone deacetylase inhibitors as anticancer agents: a review. Mol Pharmacol 68: 917-932, 2005. 
16. Darkin-Rattray SJ, Gurnett AM, Myers RW, Dulski PM, Crumley TM, Allocco JJ, Cannova C, Meinke PT, Colletti SL, Bednarek MA, Singh SB, Goetz MA, Dombrowski AW Polishook JD and Schmatz DM: Apicidin: a novel antiprotozoal agent that inhibits parasite histone deacetylase. Proc Natl Acad Sci USA 93: 13143-13147, 1996.

17. Andrews KT, Walduck A, Kelso MJ, Fairlie DP, Saul A and Parsons PG: Anti-malarial effect of histone deacetylation inhibitors and mammalian tumour cytodifferentiating agents. Int J Parasitol 30: 761-768, 2000

18. Ahn MY, Lee JW, Na YJ, Choi WS, Lee BM, Kang KW and Kim HS: Mechanism of apicidin-induced cell cycle arrest and apoptosis in Ishikawa human endometrial cancer cells. Chem Biol Interact 179: 169-177, 2009.

19. Ahn MY, Na YJ, Lee JW, Lee BM and Kim HS: Apicidin induces apoptosis via Cytochrome c-mediated intrinsic pathway in human ovarian cancer cells. Biomole \& Therape 17: 17-24, 2009.

20. Amant F, Moerman P, Neven P, Timmerman D, van Limbergen E and Vergote I: Endometrial cancer. Lancet 366: 491-505, 2005.

21. Delmonte A and Sessa C: Molecule-targeted agents in endometrial cancer. Curr Opin Oncol 20: 554-559, 2008.

22. Takai $\mathrm{N}$ and Narahara $\mathrm{H}$ : Human endometrial and ovarian cancer cells: histone deacetylase inhibitors exhibit antiproliferative activity, potently induce cell cycle arrest, and stimulate apoptosis. Curr Med Chem 14: 2548-2553, 2007.

23. Weichert W, Denkert C, Noske A, Darb-Esfahani S, Dietel M, Kalloger SE, Huntsman DG and Köbel M: Expression of class I histone deacetylases indicates poor prognosis in endometrioid subtypes of ovarian and endometrial carcinomas. Neoplasia 10 $1021-1027,2008$

24. Jiang S, Dowdy SC, Meng XW, Wang Z, Jones MB, Podratz KC and Jiang SW: Histone deacetylase inhibitors induce apoptosis in both type I and type II endometrial cancer cells. Gynecol Oncol 105: 493-500, 2007.

25. Noh JH, Song JH, Eun JW, Kim JK, Jung KH, Bae HJ, Xie HJ, Ryu JC, Ahn YM, Wee SJ, Park WS, Lee JY and Nam SW: Systemic cell-cycle suppression by Apicidin, a histone deacetylase inhibitor, in MDA-MB-435 cells. Int J Mol Med 24: 205-226, 2009.

26. Folkman $\mathrm{J}$ : What is the evidence that tumors are angiogenesis dependent? J Natl Cancer Inst 82: 4-6, 1990.

27. Kim MS, Kwon HJ, Lee YM, Baek JH, Jang JE, Lee SW, Moon EJ, Kim HS, Lee SK, Chung HY, Kim CW and Kim KW: Histones deacetylases induce angiogenesis by negative regulation of tumor suppressor genes. Nat Med 7: 437-443, 2001.

28. Kazi AA and Koos RD: Estrogen-induced activation of hypoxiainducible factor-1alpha, vascular endothelial growth factor expression, and edema in the uterus are mediated by the phosphatidylinositol 3-kinase/Akt pathway. Endocrinology 148: 2363-2374, 2007.
29. Liu F, Wang P, Jiang X, Tan G, Qiao H, Jiang H, Krissansen GW and Sun X: Antisense hypoxia-inducible factor 1alpha gene therapy enhances the therapeutic efficacy of doxorubicin to combat hepatocellular carcinoma. Cancer Sci 99: 2055-2061, 2008.

30. Garber K: HDAC inhibitors overcome first hurdle. Nat Biotechnol 25: 17-19, 2007.

31. Paris M, Porcelloni M, Binaschi M and Fattori D: Histone deacetylase inhibitors: from bench to clinic. J Med Chem 51: 1505-1529, 2008

32. Witt O, Deubzer HE, Milde T and Oehme I: HDAC family: What are the cancer relevant targets? Cancer Lett 277: 8-21, 2009.

33. Marks PA and Xu WS: Histone deacetylase inhibitors: potential in cancer therapy. J Cell Biochem 107: 600-608, 2009.

34. Weichert W, Roske A, Gekeler V, Beckers T, Ebert EP, Pross M, Dietel M, Denkert C and Rocken C: Association of patterns of class I histone deacetylase expression with patient prognosis in gastric cancer: a retrospective analysis. Lancet Oncol 9: 139-148, 2008.

35. Weichert W, Roske A, Gekeler V, Beckers T, Stephan C, Jung K, Fritzsche FR, Niesporek S, Denkert C, Dietel M and Kristiansen G: Histone deacetylases 1,2 and 3 are highly expressed in prostate cancer and HDAC2 expression is associated with shorter PSA relapse time after radical prostatectomy. Br J Cancer 98: 604-610, 2008.

36. Weichert W, Roske A, Niesporek S, Noske A, Buckendahl AC, Dietel M, Gekeler V, Boehm M, Beckers T and Denkert C: Class I histone deacetylase expression has independent prognostic impact in human colorectal cancer: specific role of class I histone deacetylases in vitro and in vivo. Clin Cancer Res 14: 1669-1677, 2008.

37. Wilson AJ, Byun DS, Popova N, Murray LB, L'Italien K, Sowa Y, Arango D, Velcich A, Augenlicht LH and Mariadason JM: Histone deacetylase 3 (HDAC3) and other class I HDACs regulate colon cell maturation and p21 expression and are deregulated in human colon cancer. J Biol Chem 281: 13548-13558, 2006.

38. Wang LG, Liu XM, Fang Y, Dai W, Chiao FB, Puccio GM, Feng J, Liu D and Chiao JW: De-repression of the p21 promoter in prostate cancer cells by an isothiocyanate via inhibition of HDACs and c-Myc. Int J Oncol 33: 375-380, 2008.

39. Chauchereau A, Mathieu M, de Saintignon J, Ferreira R, Pritchard LL, Mishal Z, Dejean A and Harel-Bellan A: HDAC4 mediates transcriptional repression by the acute promyelocytic leukaemia associated protein PLZF. Oncogene 23: 8777-8784, 2004.

40. Wilson AJ, Byun DS, Nasser S, Murray LB, Ayyanar K, Arango D, Figueroa M, Melnick A, Kao GD, Augenlicht LH, Mariadason JM: HDAC4 promotes growth of colon cancer cells via repression of p21. Mol Biol Cell 19: 4062-4075, 2008. 